
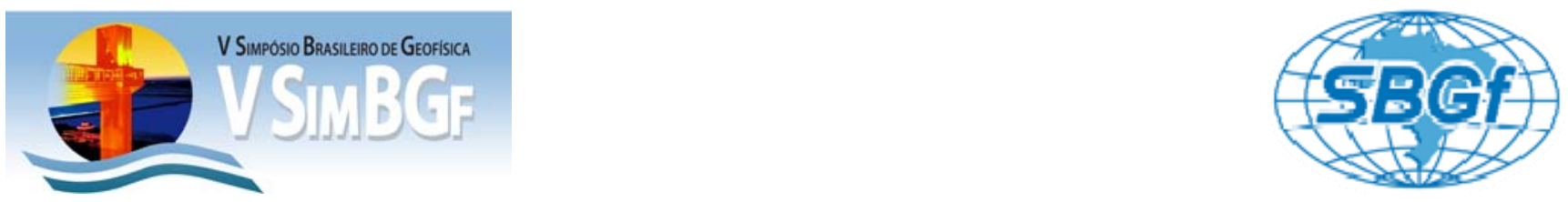

\title{
Experimento Numérico de Petrofísica e Tomografia Sísmica de Difração para o Monitoramento da Injeção de CO2.
}

Caio Jean Matto Grosso da Silva (PETROBRAS \& IGEO/UFBA), Amin Bassrei (IGEO/CPGG/UFBA)

Copyright 2012, SBGf - Sociedade Brasileira de Geofísica

Este texto foi preparado para a apresentação no V Simpósio Brasileiro de Geofísica, Salvador, 27 a 29 de novembro de 2012. Seu conteúdo foi revisado pelo Comitê Técnico do V SimBGf, mas não necessariamente representa a opinião da SBGf ou de seus associados. É proibida a reprodução total ou parcial deste material para propósitos comerciais sem prévia autorização da SBGf.

\section{Resumo}

A injeção de dióxido de carbono $\left(\mathrm{CO}_{2}\right)$ em reservatórios, para fins de aumento do fator de recuperação ou EOR (Enhanced Oil Recovery) ou para a captura e armazenamento do carbono ou CCS (Carbon Capture and Storage), tem se mostrado uma técnica empregada e bastante desejada, uma vez que possibilita o desenvolvimento sustentável de empresas petrolíferas. Após ser injetado em subsuperfície, $\mathrm{O}_{\mathrm{CO}_{2}}$ precisa ser monitorado. O objetivo deste trabalho é analisar e validar a aplicabilidade da tomografia sísmica de difração, com enfoque na geometria poço-poço ou XWP (Cross Well Profile), no monitoramento da injeção de $\mathrm{CO}_{2}$. Dado que a tomografia de difração é um problema inverso malposto, constatou-se a necessidade de um procedimento de regularização. E para a escolha do parâmetro de regularização decidiu-se utilizar a validação cruzada generalizada ou GCV (Generalized Cross Validation).

\section{Introdução}

Apesar de a sociedade humana ter opiniões divididas à respeito de se $\mathrm{o} \mathrm{CO}_{2}$ emitido pelo homem é ou não o principal responsável pelo aquecimento global, os governos têm incentivado a redução da emissão de $\mathrm{CO}_{2}$ na atmosfera. Numa perspectiva geológica e petrolífera, o $\mathrm{CO}_{2}$ pode ocorrer em conjunto com o petróleo nos reservatórios. Logo surge a questão: o que fazer com este gás?

Uma técnica que vem se tornando cada vez mais comum é a injeção de $\mathrm{CO}_{2}$ em reservatórios porosos, com duas finalidades, aumentar a recuperação (EOR) ou armazenar $\mathrm{O} \mathrm{CO}_{2}$ em aqüíferos salinos (CCS). Neste contexto, apresenta-se a tomografia de difração, que permite estimar pequenas variações de velocidade, dessa forma, pretende-se aplicá-la para monitorar o comportamento do gás dentro do reservatório.

A tomografia de difração é uma técnica não-linear e para linearizá-la, foi aplicada, neste trabalho, a aproximação de Born. Além disso, a tomografia de difração é um problema mal-posto e para aumentar o condicionamento do sistema utilizou-se a regularização por matrizes de derivadas (Tikhonov and Arsenin, 1977). E o parâmetro de regularização ótimo foi obtido através do GCV (Wahba, 1990).

Para analisar a viabilidade da tomografia de difração como ferramenta de monitoramento de injeção de $\mathrm{CO}_{2}$ em reservatórios porosos, realizou-se simulações sintéticas com dados contaminados com diferentes níveis de ruído. Para estimar qual seria a variação de velocidade devido à injeção de fluido dentro do reservatório, foi utilizado a equação de Gassmann (Gassmann, 1951).

A utilização da tomografia de difração como ferramenta para o monitoramento da injeção de $\mathrm{CO}_{2}$ já foi sugerida e empregada por vários pesquisadores. Santos et al. (2009), por exemplo, utilização essa técnica num exemplo sintético onde uma pluma de $\mathrm{CO}_{2}$ simulava um vazamento que era monitorado. Para o procedimento inverso eles empregaram o SVD juntamente com a regularização por matrizes de derivadas. Para a seleção do parâmetro de regularização foram empregadas a curva L e a curva $\Theta$. Mais recentemente, Santos et al. (2011), fizeram um experimento numérico usando tomografia de difração num modelo de camadas inclinadas inspirado no Campo de Miranga, Bacia do Recôncavo. No presente trabalho também realizou num modelo de camadas inclinadas inspirado no Campo de Miranga, sendo que a modelagem direta de velocidades levou em conta os parâmetros petrofísicos das camadas em questão.

Após a realização das simulações consideramos que o imageamento tomográfico dos reservatórios foi satisfatório, validando a aplicabilidade da tomografia sísmica de difração, com geometria poço-poço, para monitoramento da injeção de $\mathrm{CO}_{2}$ em reservatórios porosos.

\section{Problemas Inversos e Regularização}

Devido à complexidade dos estudos geofísicos é comum se trabalhar com matrizes que não são quadradas e/ou não tem posto completo, portanto, que não possuem inversa tradicional, no sentido de Cayley. Para se contornar este problema pode-se utilizar o conceito de inversa generalizada (Penrose, 1955). Para a implementação do mesmo fizemos uso da decomposição por valores singulares ou SVD (Singular Value Decomposition) que permite estimar uma matriz pseudoinversa. A técnica de SVD consiste na fatoração de uma matriz $G$ em outras três matrizes:

$$
G=U \Sigma V^{T},
$$


onde $\Sigma$ é uma matriz diagonal que possui os valores singulares da matriz $G$ dispostos em ordem crescente e as matrizes $\mathrm{U}$ e $\mathrm{V}$ são matrizes ortonormais. A partir das propriedades das matrizes supracitadas, pode-se determinar uma matriz inversa generalizada (Penrose, 1955), que no caso será expressa por:

$$
G^{+}=V \Sigma^{+} U^{T},
$$

onde $\Sigma^{+}$é, também, uma matriz diagonal que contêm os recíprocos dos valores singulares da matriz $G$. Menke (1984) afirma que os problemas inversos lineares ou linearizados podem ser formulados como um sistema linear de equações:

$$
\mathbf{d}=G \mathbf{m}
$$

onde $\mathbf{d}$ é o vetor dos parâmetros de dado, $\mathbf{m}$ é o vetor dos parâmetros de modelo e $G$ é a matriz que correlaciona os parâmetros de dado aos parâmetros de modelo. A tomografia de difração é um problema inverso mal-posto, para amenizar o problema foi escolhido obter a solução utilizando a técnica de regularização por matrizes de derivadas. Em geral essa técnica é chamada de regularização de Tikhonov (Tikhonov, 1963; Tikhonov and Arsenin, 1977). No entanto, na mesma época outros pesquisadores desenvolveram a mesma técnica em problemas discretos, tais como Phillips (1962), Cook (1963) e Twomey (1963), de modo que preferimos chamar essa técnica de regularização por matrizes de derivadas. Um breve análise da contribuição desses quatro pesquisadores pode ser vista em Bassrei and Rodi (1993).

A solução do problema inverso com regularização é expressa como

$$
\mathbf{m}=\left(G^{T} G+\lambda D_{n}^{T} D_{n}\right)^{-1} G^{T} \mathbf{d},
$$

onde $D_{n}$ é a matriz de derivadas de ordem $n$ e $\lambda$ é o parâmetro de regularização. A escolha do parâmetro é problema específico e existem na literatura várias alternativas, tais como a curva $L$, reintroduzida $e$ popularizada por Hansen (1992). A curva L e uma extensão do mesmo chamada de curva $\Theta$ foram utilizada em várias aplicações de tomografia, seja tomografia de difração (Santos and Bassrei, 2007a; Santos et al. 2009). Para estimar qual o parâmetro de regularização ótimo utilizou-se um outro critério, chamado de validação cruzada generalizada ou GCV (Generalized Cross Validation), desenvolvido por $\mathrm{G}$. Wahba na década de 1970, que pode ser encontrado no seu livro (Wahba, 1990). Para a escolha do parâmetro de regularização $\lambda$ é necessário localizar o mínimo da função de validação cruzada, expressa como:

$$
V(\lambda)=\frac{\left\|\mathbf{d}^{o b s}-\mathbf{d}\left(m_{\lambda}\right)\right\|}{\frac{1}{M} \operatorname{Tr}[I-A(\lambda)]}
$$

onde $A(\lambda)$ é uma matriz auxiliar expressa como

$$
A(\lambda)=G\left(G^{T} G+\lambda D_{n}^{T} D_{n}\right)^{-1} G^{T} .
$$

O GCV foi utilizado em tomografia de difração por Santos and Bassrei (2007b).

\section{Tomografia Sísmica de Difração}

A onda elástica tem propriedades de difração, pois quando esta se propaga através dum meio e encontra objetos que tenham as mesmas dimensões que o seu comprimento de onda sua energia é difratada. Logo a partir do espalhamento da onda é possível inferir qual a velocidade do meio.

A propagação de uma determinada perturbação através de um meio com densidade constante e uma variação de velocidade $c(r)$ pode ser descrita, no domínio da frequência, através da equação de Helmholtz:

$$
\nabla^{2} P(r, \omega)+\kappa^{2}(r, \omega) P(r, \omega)=0,
$$

sendo $P(r, \omega)$ a amplitude da onda definida em função da frequência angular e $\kappa(r, \omega)$ o número de onda, que é definido como a razão entre a frequência angular e a velocidade do meio.

O problema do espalhamento consiste em uma onda incidente $P_{i}(r)$ que se propaga a partir da fonte num meio com velocidade de background $C_{0}$. Pretende-se imagear uma estrutura de área $\boldsymbol{A}$ com contraste de velocidade $C(r)$ em relação à velocidade de background, que atua como uma fonte secundária e espalha o campo de onda incidente. O campo de onda registrado por um receptor consiste da onda incidente e da onda espalhada, chamada de campo de onda total:

$$
P_{t}=P_{i}+P_{s} \text {, }
$$

onde $P_{s}$ é o campo espalhado. Definindo uma função objetivo como:

$$
M(r)=\left(1-\frac{c_{0}^{2}}{c^{2}(r)}\right)
$$

Lo and Inderwiesen (1994), apresentam a equação de Lippmann-Schwinger, que relaciona não linearmente a função de dados $P_{s}$ com a função de modelo $M(r)$ :

$$
P_{s}=-\kappa_{0}^{2} \int_{A} G\left(r \mid r^{\prime}\right) M\left(r^{\prime}\right)\left[P_{i}\left(r^{\prime}\right)+P_{s}\left(r^{\prime}\right)\right] d r^{\prime} \text {. }
$$

Esta equação apresenta uma relação de não linearidade que reside no fato do campo espalhado estar presente dentro da integral. Este problema pode ser contornado utilizando a aproximação de Born que assume que o campo de onda espalhado é muito menor que o incidente, sendo, portanto, desprezado o seu valor dentro da integral da equação (10). 


\section{Equação de Gassmann}

A equação de Gassmann (Gassmann, 1951) é uma ferramenta valiosa no estudo de reservatórios, pois permite a possibilidade de se modelar vários cenários com diferentes tipos e saturações de fluidos. A equação relaciona o módulo de incompressibilidade de uma rocha através das propriedades dos poros, arcabouço e fluidos:

$$
K_{\text {sat }}=K_{\mathrm{sec} a}+\frac{\left(1-\frac{K_{\mathrm{sec} a}}{K_{\text {min }}}\right)^{2}}{\frac{\phi}{K_{f l}}+\frac{(1-\phi)}{K_{\text {min }}}-\left(\frac{K_{\mathrm{sec} a}}{K_{\text {min }}}\right)^{2}},
$$

onde $K_{\text {sat }}$ é o módulo de incompressibilidade de uma rocha saturada com um fluido de módulo de incompressibilidade $K_{f l}, K_{\text {seca }}$ é o módulo de incompressibilidade da rocha seca, $K_{\text {min }}$ é o módulo de incompressibilidade do mineral que compõem a rocha e, $\phi$ é a porosidade. Logo, com a equação de Gassmann, pode-se estimar qual será a variação de velocidade devido à mudança de fluidos dentro de um reservatório.

\section{Resultados}

Para avaliar o potencial da tomografia de difração como método de monitoramento de injeção de $\mathrm{CO}_{2}$, fez-se algumas simulações numéricas. Para tanto, se idealizou três estágios: o primeiro onde o aquífero salino estava completamente saturado de água e dois estágios subseqüentes à injeção de $\mathrm{CO}_{2}$.

A Figura 1 ilustra o modelo no seu estado inicial. Para a realização das operações de simulação o meio foi discretizado em uma malha $30 \times 30$, totalizando 900 blocos. Como geometria de aquisição utilizou-se a interpoços, onde foram colocadas 15 fontes no poço à esquerda e 30 receptores no poço à direita. Como a tomografia de difração lida com números complexos, é necessário fazer a decomposição do campo espalhado nas suas partes real e imaginária. Desse modo, há um aumento de 900 valores de parâmetros de dado para cada frequência utilizada. O trabalho fez uso de uma abordagem multifrequência, onde as freqüências utilizadas foram: 90, 105, 120 e $135 \mathrm{~Hz}$, portanto, a matriz sensibilidade que relaciona os parâmetros de dados aos parâmetros de modelo é da ordem de 3600 x 900.

O modelo apresenta três camadas paralelas de arenito (nas cores azul escuro, amarelo e marrom) seladas por folhelhos de baixa impedância (azul claro).

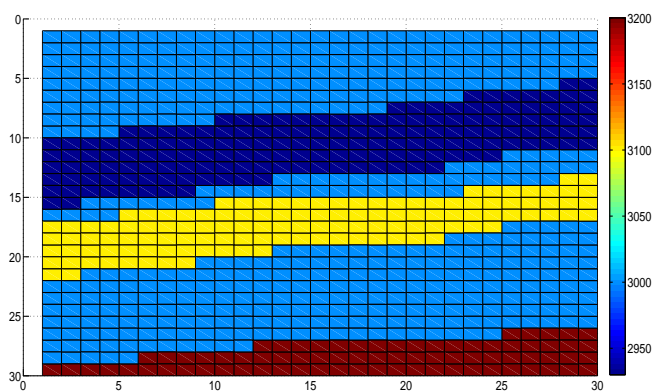

Figura 1 - Modelo verdadeiro para o estágio 1.

A Tabela 1 ilustra as propriedades dos reservatórios nos três estágios que serão considerados.

\begin{tabular}{|l|l|l|l|l|}
\hline$S_{\mathrm{H}_{2} \mathrm{O}}$ & $S_{\mathrm{CO}_{2}}$ & $K_{\text {sat }}$ & $\rho_{\text {sat }}$ & $V_{P}$ \\
\hline $100 \%$ & $0 \%$ & $12.8 \mathrm{GPa}$ & $2.14 \mathrm{~g} / \mathrm{cm} 3$ & $2930 \mathrm{~m} / \mathrm{s}$ \\
\hline $70 \%$ & $30 \%$ & $8.85 \mathrm{GPa}$ & $2.11 \mathrm{~g} / \mathrm{cm} 3$ & $2610 \mathrm{~m} / \mathrm{s}$ \\
\hline $40 \%$ & $60 \%$ & $8.81 \mathrm{GPa}$ & $2.09 \mathrm{~g} / \mathrm{cm} 3$ & $2560 \mathrm{~m} / \mathrm{s}$ \\
\hline
\end{tabular}

Tabela 1 - Propriedades do reservatório.

O módulo de incompressibilidade e a densidade para o $\mathrm{CO}_{2}$ e a água utilizados foram, respectivamente, 0.25 GPa, 2,25 GPa, $0.71 \mathrm{~g} / \mathrm{cm} 3$ e $1 \mathrm{~g} / \mathrm{cm} 3$.

A Figura 2 apresenta o comportamento da GCV com diferentes níveis de ruído em função dos parâmetros de regularização testado.

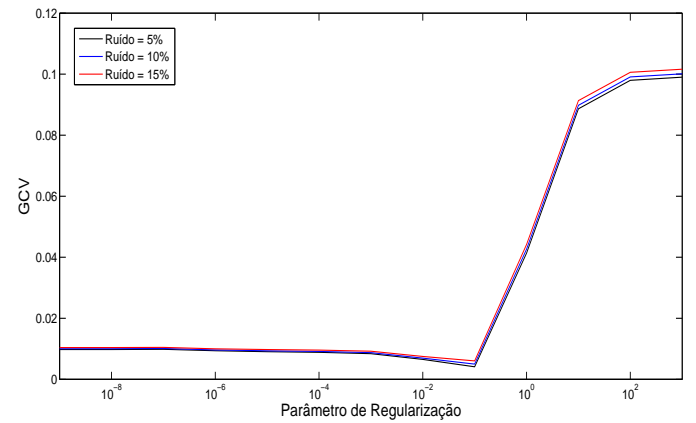

Figura 2 - GCV para diferentes níveis de ruído no estágio 1.

A partir da análise da Figura 2 pode-se concluir que 0 parâmetro ótimo é de $10^{-1}$. A Figura 3 apresenta o modelo estimado com o parâmetro de regularização ótimo. 


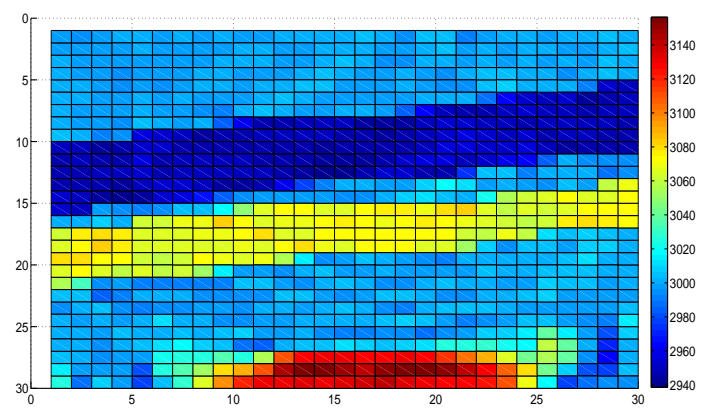

Figura 3 - Modelo estimado para o estágio 1.

A Figura 4 apresenta o modelo idealizado para o estágio 2 , onde o reservatório está agora divido em duas zonas de diferentes saturações de água, zona 1 com saturação de $100 \%$ e zona 2 com saturação de $70 \%$, os valores de velocidades pode ser verificado na Tabela 1.

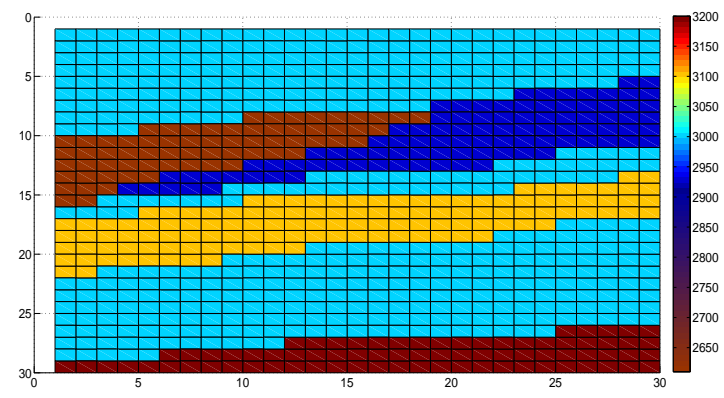

Figura 4 - Modelo verdadeiro para o estágio 2.

A Figura 5 ilustra o comportamento da GCV para diferentes níveis de ruído.

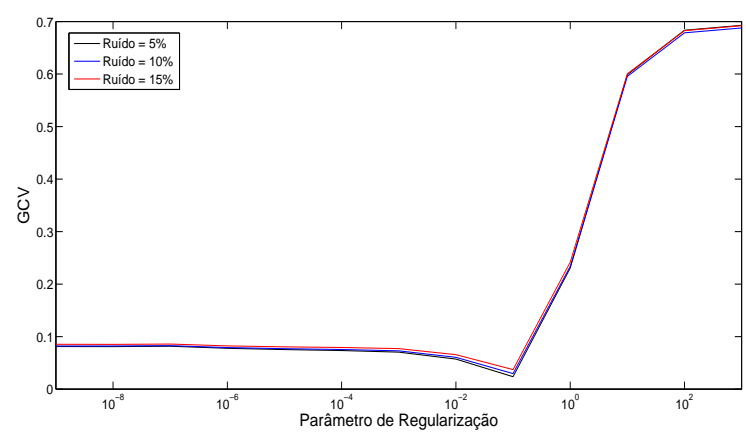

Figura 5 - GCV para diferentes níveis de ruído no estágio 2.

A Figura 6 apresenta o modelo estimado para o 2 estágio utilizando o parâmetro ótimo $\lambda=10^{-1}$.

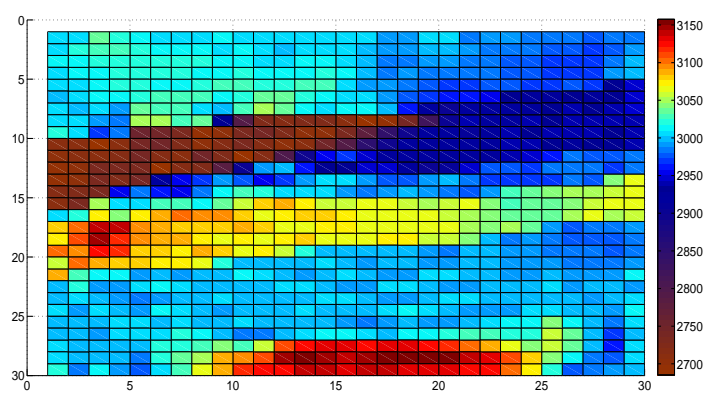

Figura 6 - Modelo estimado para o estágio 2.

Para avaliar o comportamento do $\mathrm{CO} 2$ dentro do reservatório calculou-se a diferença entre os modelos, estimados, dos estágios 1 e 2. A Figura 7 apresenta o modelo residual.

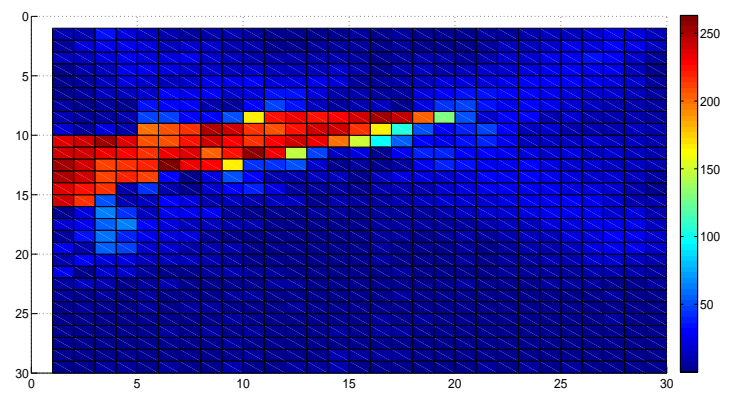

Figura 7 - Modelo residual entre os estágios 1 e 2 .

Por fim, idealizou-se o terceiro estágio, onde o reservatório de injeção foi compartimentado por três níveis de saturação distintos. A Figura 8 apresenta o modelo verdadeiro, onde a cor cinza indica saturação de água igual a $40 \%$, a cor marrom com saturação de água igual a $70 \%$ e a cor azul escuro com a saturação original de $100 \%$.

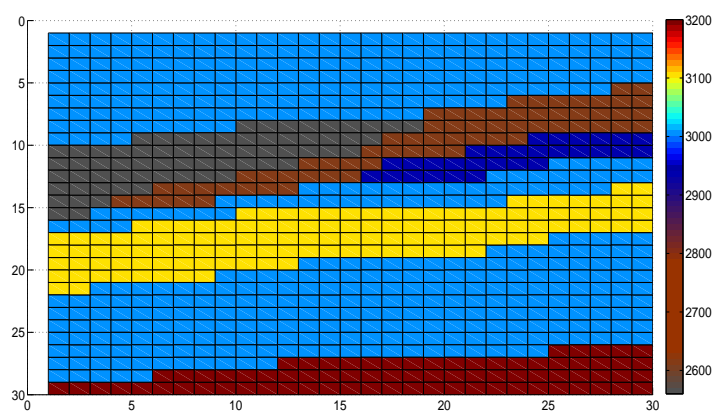

Figura 8 - Modelo verdadeiro para o estágio 3.

Novamente, para obter o parâmetro ótimo de regularização, gerou-se a curva de GCV apresentada na Figura 9. 


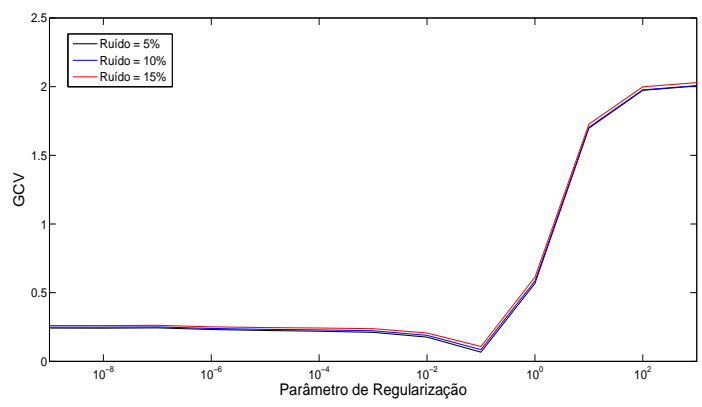

Figura 9-GCV para diferentes níveis de ruído no estágio 3.

A Figura 10 mostra o modelo estimado para o derradeiro estágio considerado com o parâmetro de regularização ótimo $\lambda=10^{-1}$.

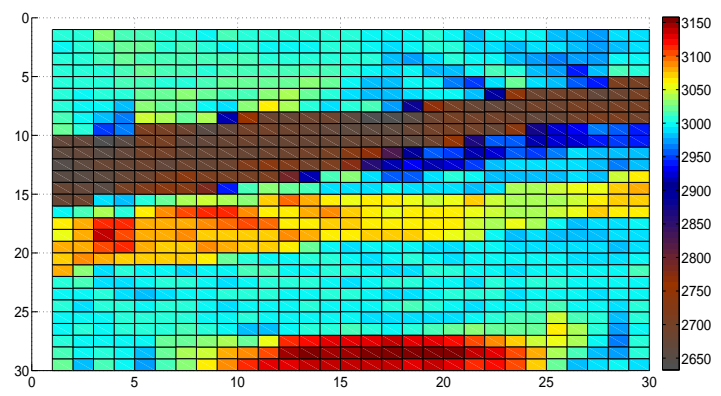

Figura 10 - Modelo estimado para o estágio 3.

As Figuras 11 e 12 apresentam, respectivamente, os modelos residuais entre os estágios 1 e 3 , e os estágios 2 e 3.

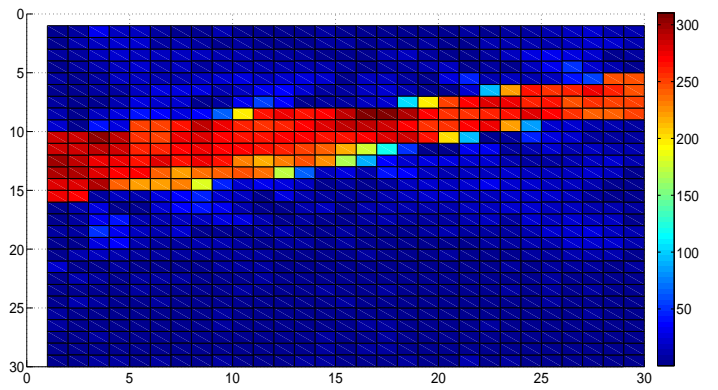

Figura 11 - Modelo residual entre os estágios 1 e 3 .

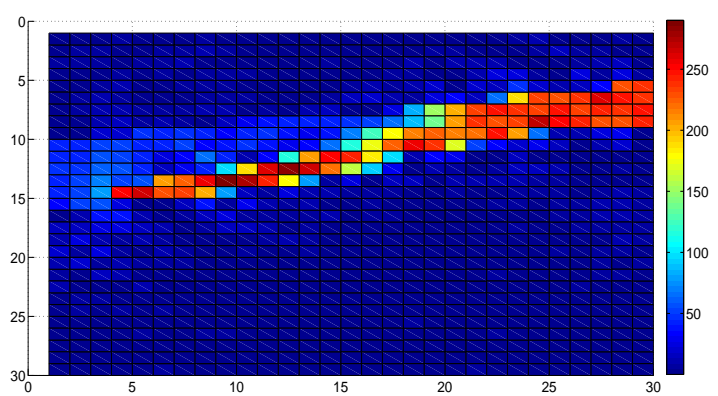

Figura 12 - Modelo residual entre os estágios 2 e 3 .

\section{Discussão e Conclusões}

A partir da equação de Gassmann foi possível estimar qual seria a mudança de velocidade dentro de um reservatório devido à substituição de fluidos e, dessa forma, se analisar a viabilidade da tomografia de difração para monitoramento sísmica de injeção de fluidos em reservatórios arenosos.

A tomografia sísmica de difração, com uma abordagem multifrequência, se mostrou uma ferramenta robusta no monitoramento de injeção de $\mathrm{CO} 2$ em reservatórios arenosos, uma vez que foi possível identificar a migração do fluido no reservatório devido às variações de velocidades percebidas nos diversos estágios. Além disso, os valores de velocidade estimados, mas claramente mostrados nos modelos residuais (Figuras 7 , 11 e 12), apresentaram-se coerentes com aqueles valores de velocidades modelados com a equação de Gassmann.

O método de regularização por matrizes de derivadas se mostrou adequado à melhoria do condicionamento do problema inverso, permitindo estimar soluções bastante próximas das verdadeiras. Ainda, a GCV se mostrou uma ferramenta robusta na escolha do parâmetro ótimo de regularização. Entretanto, coincidentemente os valores dos parâmetros de regularização foram de $\lambda=10^{-1}$, mas este parâmetro pode variar de acordo com a estabilidade do sistema. Além disso, apesar do parâmetro de regularização ser o mesmo para os diferentes estágios os valores do GCV foram distintos para os mesmos.

Por fim, pode-se afirmar, de maneira geral, que a tomografia de difração, com uma geometria poço-poço, é uma ferramenta válida para o monitoramento da injeção de $\mathrm{CO}_{2}$. Mas, devido as mais variadas condições e propriedades de reservatórios, deve-se, invariavelmente, realizar um estudo de viabilidade antes do projeto de monitoramento.

\section{Agradecimentos}

Os autores agradecem à PETROBRAS pelo financiamento do projeto Aplicação da Tomografia Sísmica para o Monitoramento do Armazenamento Geológico de $\mathrm{CO}_{2}$. Caio Silva agradece ao $\mathrm{CNPq}$ pela bolsa de mestrado e Amin Bassrei agradece ao CNPq pela bolsa de produtividade de pesquisa. Os autores agradecem ao CNPq (INCT - Geofísica de Petróleo) e à FINEP (Rede CT-PETRO em Geofísica de Exploração) pelo financiamento e apoio à pesquisa. 


\section{Referências}

Bassrei, A. and Rodi, W. L., 1993, Regularization and inversion of linear geophysical data. In: Proceedings of the Third International Congress of the Brazilian Geophysical Society, Rio de Janeiro, Brazil, vol. 1, pp. 111-116.

Cook, B. C., 1963, Least structure analysis of photonuclear yield functions, Nuclear Instruments and Methods, vol 24. 256-268.

Gassmann, F., 1951, Über die Elastizität poröser Medien: Vierteljahrschrift der Naturforschenden Gesellschaft in. Zürich, 96, 1-23.

Hansen, P. C., 1992, Analysis of discrete ill-posed problems by means of the L-curve. Society for Industrial and Applied Mathematics Review, vol. 34, 561-580.

Lo, T. W. and Inderwiesen, O. L., 1994, Fundamentals of Seismic Tomography. Geophysical Monograph Series, Tulsa, OK, 178pp.

Menke, W., 1984, Geophysical Data Analysis: Discrete Inverse Theory, Academic Press, Orlando, FL.

Penrose, R., 1955, A Generalized Inverse for Matrices, Proceedings of the Cambridge Philosophical Society, vol. 51, 406-413.

Phillips, D. L., 1962, A technique for the numerical solution of certain integral equations of the first kind, Journal of Association of Computing Machinery, vol. 9, 84-97.

Santos, E. T. F. and Bassrei, A., 2007a, L-curve and $\Theta-$ curve approaches for the selection of regularization factor in geophysical diffraction tomography, Computers \& Geosciences, vol. 33, p. 618-629.

Santos, E. T. F. and Bassrei, A., 2007b. Application of GCV in geophysical diffraction tomography. 69th European Association of Geoscientists and Engineers Conference \& Exhibition, London, CD-ROM, 5 pp.

Santos, E. T. F., Harris, J. M., Bassrei, A. and Costa, J. C., 2009, Trigonal meshes in diffraction tomography with optimum regularization: an application for carbon sequestration monitoring, Journal of Seismic Exploration, vol. $18,135-156$.

Santos, E. T. F., Bassrei, A., Harris. J. M. e Costa, J. C., 2011, Tomografia de difração aplicada ao problema do armazenamento geológico do carbono, $1^{\circ}$ Congresso Brasileiro de CO2 na Indústria de Petróleo, Gás e Biocombustíveis, 18 a 20 de abril de 2011, resumo IBP176-11.

Tikhonov, A. N. and Arsenin V. Y., 1977, Solution of IIIPosed Problems, Wiley, Washington.
Tikhonov, A. N., 1963, Solution of incorrectly formulated problems and the regularization method, Soviet Mathematics, vol. 4, 1035-1038.

Twomey, S., 1963, On the numerical solution of Fredholm integral equation of the first kind by the inversion of the linear system produced by quadrature, Journal of Association of Computing Machinery, vol. 10, 97-101.

Wahba, G., 1990, Spline Models for Observational Data. SIAM, Philadelphia. 